Skin Symptoms Limit Their Ability to Participate in Activities, Causes Chronic Physical Discomfort, and Can Negatively Impact Self-Esteem

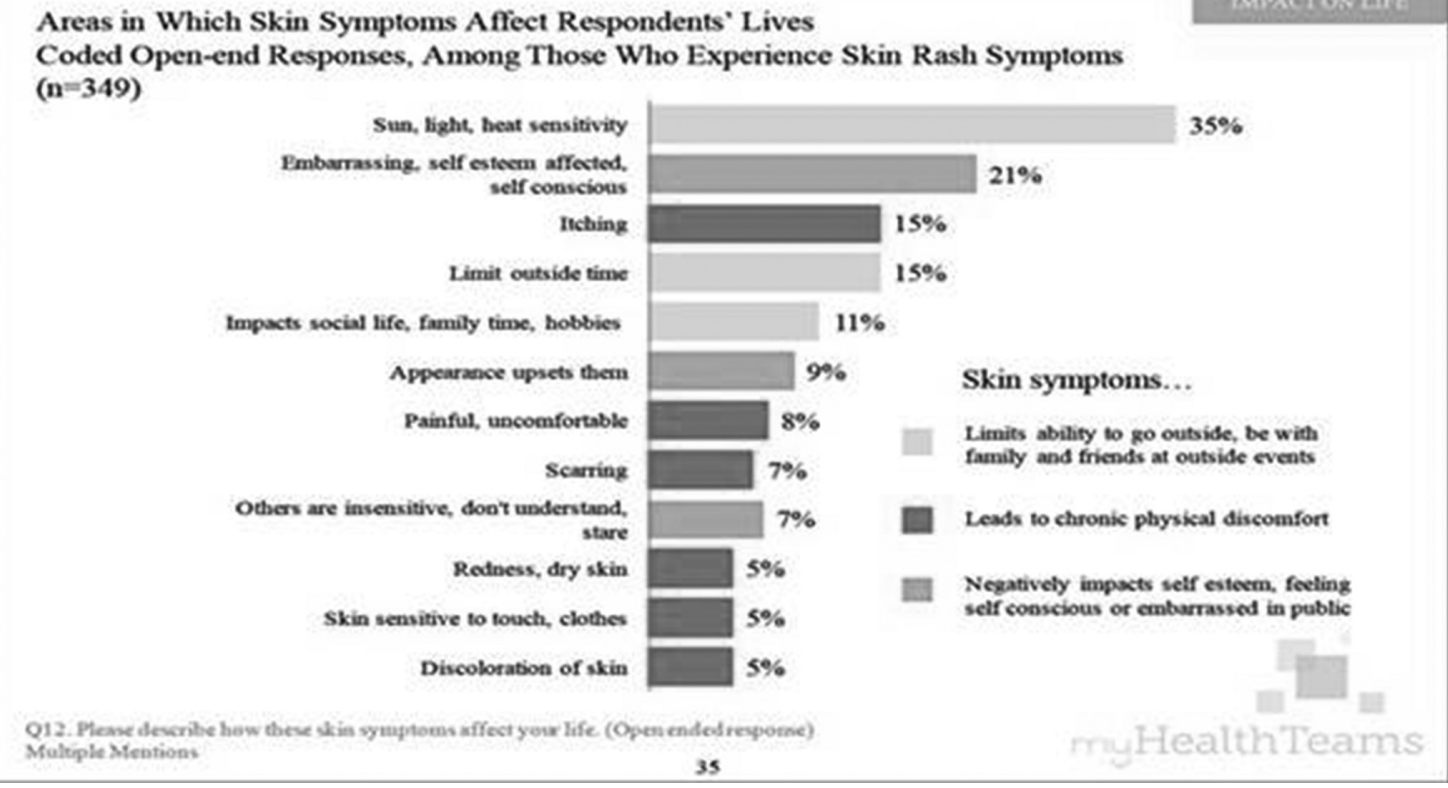

Abstract 162 Figure 2

( $\geq 1.6 \mathrm{mg} / \mathrm{dL}$ for $\mathrm{men}, \geq 1.4 \mathrm{mg} / \mathrm{dL}$ for women) were identified from medical record. This study used the data in time the patients were diagnosed. The data of SLE patients from 2008 to 2016 were recorded in RSHS Lupus Registry. Chi-square analyses was performed to determine the association between those variabels.

Results A total of 428 SLE patients had a median age of 35 years (97.9\% female), 64 of them (15\%) were hypertensive, 176 SLE patients (41.1\%) had proteinuria, and 106 SLE patients $(24.8 \%)$ had elevated serum creatinine level. Forty one SLE patients with hypertension (64.1\%) had proteinuria. Hypertension was associated with proteinuria in SLE patients (95\% CI, Pearson Chi-Square 18.948, asymptotic significance $<0.001)$. Elevated serum creatinine level had no association with hypertension (95\% CI, Pearson Chi-Square 0.071, asymptotic significance 0.789 ) and with proteinuria (95\% CI, Pearson Chi-Square 0.603 , asymptotic significance 0.438 ).

Conclusions In this study, hypertension is associated with proteinuria. There are no associations between hypertension and proteinuria with elevated serum creatinine level.

\section{SJOGREN'S SYNDROME AND LOCALISEDLOCALIZED NODULAR CUTANEOUS AMYLOIDOSIS: NEW INSIGHTS INTO THE LINK BETWEEN THE TWO}

${ }^{1}$ N Walsh*, ${ }^{2}$ I.M. Lano, ${ }^{3}$ J.G. Hanly, ${ }^{4}$ L Cerroni. ' Dalhousie University, Pathology, Halifax, Canada; ${ }^{2}$ Nova Scotia Health Authority, Pathology, Halifax, Canada; ${ }^{3}$ Dalhousie University, Rheumatology, Halifax, Canada; ${ }^{4}$ Medical University of Graz, Dermatology, Graz, Austria

\subsection{6/lupus-2017-000215.164}

Background and aims Sjogren's Syndrome (SS), a known complication of systemic lupus erythematosus, is associated with localised nodular cutaneous amyloidosis, AL type (AL-LNCA).
The reason is unclear, but clues from studies of this rare variant of amyloidosis are emerging.

Methods Six patients with AL-LNCA, 4 from Austria and 2 from Canada were identified. Clinical, demographic and histopathological data were recorded and outcome noted over a median period of 72 months (range 40-144).

Results Of 3 men and 3 women (median age 57 years; range 36-72) 1 patient had diabetes mellitus and essential hypertension and another scleroderma. The skin lesions were tan plaques or nodules, $1.5-4.0 \mathrm{~cm}$ in size, on the legs (5) and arm (1). Microscopically, bulky deposits of AL amyloid in the dermis/subcutis were associated with light perivascular infiltrates of lymphocytes and monoclonal plasma cells (with kappa (3) or lambda (3) light chain restriction). Two patients developed local cutaneous recurrences of their AL-LNCA 4 and 5 years after presentation. None developed systemic amyloidosis.

Conclusions The clinical phenotype and course of AL-LNCA in our series, like those in the literature, mirror those of primary cutaneous marginal zone lymphoma, lymphoplasmacytic variant. This is now included among the larger group of extranodal B-cell lymphomas of MALT. Patients with SS are at risk for the development of MALT lymphomas. These, in turn, are known to be associated with localised peritumoral amyloidosis in internal organs. We submit that AL-LNCA in SS is a manifestation of a MALT lymphoma in the skin.

\section{CORRELATION BETWEEN PLASMA LEVELS OF TNF- $\alpha$ AND CAROTID ARTERY INTIMA MEDIA THICKNESS IN SLE}

RY Hellmi. Kariadi Hospital, Rheumatology, Semarang, Indonesia

10.1136/lupus-2017-000215.165 